\title{
Consultoria em amamentação durante a pandemia COVID-19: relato de experiência
}

\author{
Breastfeeding consultancy during the COVID-19 pandemic: experience report \\ Asesoría en lactancia materna durante la pandemia de COVID-19: informe de experiencia
}

Ana Carolina Maria Araújo Chagas
Costa Lima ${ }^{1}$ (B)

Anne Fayma Lopes Chaves ${ }^{2}$ (D)

Mariana Gonçalves de Oliveira ${ }^{3}$ (D)

Sabrina Alapenha Ferro Chaves

Costa Lima ${ }^{3}$ (1)

Márcia Maria Tavares Machado ${ }^{4}$ [C]

Mônica Oliveira Batista Oriá ${ }^{1}$

1. Centro Universitário Estácio do Ceará.

Fortaleza, CE, Brasil.

2. Universidade da Integração Internacional da Lusofonia Afro-Brasileira, Programa de Pós-Graduação em Enfermagem. Redenção, CE, Brasil.

3. Faculdade IDE, AMA Consultoria Materno-Infantil. Fortaleza, CE, Brasil.

4. Universidade Federal do Ceará, Programa de Pós-Graduação em Saúde Coletiva. Fortaleza, CE, Brasil.

5. Universidade Federal do Ceará, Programa de Pós-Graduação em Enfermagem. Fortaleza, $\mathrm{CE}$, Brasil.

\section{Resumo}

Objetivo: relatar a experiência de consultoras em amamentação no atendimento às lactantes durante a pandemia de COVID-19. Método: relato de experiência de atendimentos de consultoria em amamentação, nas modalidades online e presencial, na cidade de Fortaleza-CE, Brasil, entre março e abril de 2020. Resultados: diante de sentimentos de medo e ansiedade das lactantes as consultoras prestaram apoio por meio de aconselhamento, bem como orientações com equipe multiprofissional. Todas as lactantes estavam amamentando e realizando distanciamento social e cuidados de higiene pessoal, sendo reforçado pelas consultoras a importância do acesso às fontes confiáveis de informação. Quanto aos problemas mamários, foram realizadas intervenções preventivas e curativas. Percebeu-se a satisfação das lactantes pelo serviço. Conclusão e implicações para a prática: a consultoria em amamentação constitui-se como um dispositivo agregador na saúde das mulheres que favorece tanto a promoção do aleitamento materno quanto a saúde mental durante a pandemia de Covid-19. Este relato traz direcionamentos para uma prática holística, com vistas à melhoria da qualidade do cuidado prestado, levando-se em consideração os atuais desafios da promoção da saúde diante da pandemia e pode fomentar novas estratégias exitosas.

Palavras-chave: Aleitamento Materno; Infecções por Coronavírus; Enfermagem; Consultores; Saúde Mental.

\section{Abstract}

Objective: to report the experience of breastfeeding consultants in caring for breastfeeding women during the Covid-19 pandemic. Method: an experience report of breastfeeding consultancy services, online and face-to-face modalities, in the city of Fortaleza-CE, between March and April 2020. Results: given the mothers' feelings of fear and anxiety, the consultants provided support through counseling, as well as guidance with a multidisciplinary team. All the women were breastfeeding and performing social distancing and personal hygiene care actions, the importance of access to reliable information sources being reinforced by the consultants. As for breast problems, preventive and curative interventions were carried out. The mothers' satisfaction with the consultant service was noticed. Conclusion and implications for practice: breastfeeding consultancy is an aggregating device in women's health that favors both the promotion of breastfeeding and mental health during the Covid-19 pandemic. This report provides directions for a holistic practice, to improve the provided care quality, considering the current challenges of health promotion given the pandemic, and can foster new successful strategies.

Keywords: Breastfeeding; Coronavirus Infections; Nursing; Consultants; Mental Health.

\section{REsumen}

Objetivo: relatar la experiencia de consultores de lactancia materna en el cuidado de mujeres en periodo de lactancia durante la pandemia de Covid-19. Método: informe de experiencia de los servicios de consultoría en lactancia materna en modalidades en línea y cara a cara, en la ciudad de Fortaleza-CE, entre marzo y abril de 2020. Resultados: ante los sentimientos de miedo y ansiedad de las mujeres lactantes, los consultores proporcionaron apoyo a través de asesoramiento, así como orientación con un equipo multidisciplinario. Todas las mujeres amamantaban y practicaban el distanciamiento social y cuidados de higiene personal, siendo que los consultores reforzaron la importancia del acceso a fuentes confiables de información. En cuanto a los problemas mamarios, se realizaron intervenciones preventivas y curativas. Se notó la satisfacción de las madres lactantes por el servicio. Conclusión e implicaciones para la práctica: la consultoría sobre lactancia materna es un dispositivo agregado en la salud de la mujer que favorece tanto la promoción de la lactancia materna como la salud mental frente a la pandemia de Covid-19. Este estudio proporciona instrucciones para una práctica holística, con miras a mejorar la calidad de la atención brindada, teniendo en cuenta los desafíos actuales de la promoción de la salud frente a la pandemia y puede fomentar nuevas estrategias exitosas.

Palabras clave: Lactancia Materna; Infecciones por Coronavirus; Enfermería; Consultores; Salud Mental.

\section{Autor correspondente:}

Ana Carolina Maria Araújo Chagas Costa Lima. E-mail: anacarolinamaccl@gmail.com.

Recebido em 21/08/2020.

Aprovado em 23/10/2020.

DOI:https://doi.org/10.1590/2177-9465-EAN-2020-0350 


\section{INTRODUÇÃO}

A consultoria em amamentação foi regulamentada nos Estados Unidos na década de 1980, por meio do International Board of Lactation Consultant Examiners (IBLCE). O consultor em amamentação é um profissional reconhecido em vários países, possui atividade remunerada, segue um código de ética com condutas clínicas baseadas em evidências e atua de maneira bem definida em diversos ambientes: clínicas privadas, atendimento domiciliar e/ou hospitalar, unidades de terapias intensivas neonatais, bancos de leite, ambulatórios e em comunidades; utilizando técnicas de manejo clínico da lactação, com demonstrações e observações, bem como a contribuição por meio de educação em saúde. ${ }^{1}$

Assim, destaca-se a importância do papel exercido pelo consultor em lactação na promoção, proteção e apoio à amamentação, influenciando diretamente nos índices de aleitamento materno (AM). ${ }^{2}$ Ademais, verifica-se a escassez de estudos nacionais sobre o trabalho desse consultor. Entre os profissionais que atuam na área, os enfermeiros prestam assistência à mulher durante o ciclo gravídico-puerperal e possuem respaldo legal de seu conselho de classe, tendo formação superior e capacidade técnico-científica para exercer essa assistência de maneira qualificada e ética. ${ }^{3}$

Com o advento do novo Coronavírus (Covid-19) e do grave problema de saúde mundial, mais de 15 milhões de pessoas em todo o mundo já foram infectadas pela Covid-19, o que ocasionou 630.750 mortes, sendo declarada pela Organização Mundial de Saúde (OMS) como uma emergência global de saúde pública. ${ }^{4}$

Ainda não há evidência consolidada da transmissão vertical do SARSCoV-2. Embora já tenham sido evidenciados alguns sinais de alterações placentárias decorrentes de processos inflamatórios cuja suspeita recaem sobre o Covid- 19,5 o vírus ainda não foi encontrado nas amostras de líquido amniótico, cordão umbilical, swab da orofaringe de neonatos e no leite materno. ${ }^{6}$

Assim, não há evidência científica robusta que comprove a relação entre a transmissão do SARSCoV-2 e a amamentação, ${ }^{6}$ razão pela qual a maioria das diretrizes neonatais não contraindica a amamentação de mães com Covid-19, tendo em vista que os benefícios superam quaisquer riscos potenciais de transmissão do vírus a partir do leite materno. No entanto, é necessário tomar precauções específicas, como uso de máscara durante a amamentação e medidas de higiene das mãos. ${ }^{7}$

Diante desse panorama de incertezas e da pandemia Covid-19, as lactantes estão mais sujeitas ao aumento de alterações emocionais frente às inseguranças do cenário vigente. Desse modo, problemas de saúde mental podem ser agravados exigindo dos profissionais de saúde suporte contínuo e especializado. Destarte, destaca-se a importância dos consultores da amamentação que, dentro de suas possibilidades, devem dar suporte às lactantes e, nesse sentido, deve-se procurar estratégias de acompanhamento à distância, como teleconsultas e ações educativas nas mídias sociais, entre outras. ${ }^{8}$

A partir desse cenário, surgiu o seguinte questionamento: como os consultores em amamentação podem contribuir para minimizar os efeitos da pandemia Covid-19 na saúde física e mental das lactantes? Logo, apresenta-se este relato com o objetivo de descrever as experiências de consultoras em amamentação durante a pandemia Covid-19. Este relato propõe algumas estratégias que merecem ser socializadas no intuito de favorecer que outros profissionais se sensibilizem para a promoção da saúde materno-infantil, proporcionando uma atenção qualificada, que envolva o apoio emocional.

\section{DESCRIÇÃO DA EXPERIÊNCIA}

Trata-se de um relato de experiência de atendimentos de consultoria em amamentação de uma instituição atuante na cidade de Fortaleza-Ceará, Brasil. O relato de experiência consiste em um importante narrativo científico consoante à condição pós-moderna, a qual tem o poder de circunscrever a experiência, o local e seu tempo histórico, de modo articulado a um robusto conteúdo teórico. ${ }^{9}$

A consultoria acontece com atendimentos agendados a gestantes e puérperas, orientando e intervindo no processo do aleitamento materno e nos cuidados com o bebê. Além disso, ela também promove cursos para gestantes e treinamentos para profissionais, a fim de prepará-los seguindo as melhores evidências científicas.

Os atendimentos foram realizados por duas consultoras, enfermeiras e sócias da empresa, as quais são autoras do presente estudo, no período 20 de março a 20 de abril de 2020 , com 37 lactantes. Diante da recomendação do distanciamento social, 27 atendimentos foram realizados online, por meio dos aplicativos WhatsApp e Instagram, sendo utilizadas vídeo-chamadas como forma de visualizar a dinâmica da amamentação, bem como buscar maior proximidade e vínculo do binômio mãe-bebê. No entanto, nos casos que se fizeram necessários, foram realizados 10 atendimentos por meio de visitas domiciliares, sendo utilizados todos os cuidados recomendados para a prevenção da transmissão do Covid-19. A idade das lactantes atendidas variou entre 25 e 38 anos, todas com mais de 12 anos de estudo, com filhos na idade entre seis dias e um ano e três meses, que estavam amamentando.

Foram reduzidos os atendimentos realizados por dia, devido à exposição e à necessidade de tempo para que as consultoras, entre uma visita e outra, se dirigissem aos seus domicílios para realizarem higiene pessoal e limpeza dos materiais utilizados. Nos atendimentos presenciais as consultoras se paramentavam utilizando os seguintes equipamentos: avental descartável, propé, gorro descartável, máscara N-95, óculos de proteção individual e a máscara face shield.

Antes de fazer a visita domiciliar, as lactantes foram orientadas quanto à permanência de apenas um acompanhante durante o atendimento, e questionadas sobre possíveis sintomas gripais dela e dos contatos domiciliares. Ao chegar no domicílio, as consultoras deixavam os sapatos fora da residência, realizavam a desinfecção dos acessórios utilizados no atendimento com álcool a 70\% e a higienização das mãos. 
Durante o atendimento, as consultoras orientavam sobre a prática da amamentação, apoiavam e incentivavam a adesão ao AM, esclareciam as dúvidas, e enfatizavam as soluções das dificuldades expostas por cada mãe. No entanto, diante da pandemia Covid-19, percebeu-se que grande parte das lactantes se mostraram ansiosas, relatando medo e buscando informações sobre os cuidados com ela e com o bebê.

Perante esse anseio das lactantes, as consultoras decidiram enfatizar alguns cuidados no que se refere à infecção do Covid-19, tais como: apoio aos sentimentos negativos diante do processo de AM e a pandemia de Covid-19 e orientações sobre medidas realizadas para a prevenção e possíveis mudanças na amamentação. Para realizar as orientações, as consultoras utilizaram as recomendações do Ministério da Saúde (MS), da Sociedade Brasileira de Pediatria (SBP) e da Rede Brasileira de Bancos de Leite Humano (rBLH).

Devido ao fato de este estudo se constituir um relato de experiência, não foi submetido à avaliação de Comitê de Ética. No entanto, durante seu desenvolvimento, foram considerados os preceitos éticos presentes nas normativas de pesquisas científicas que envolvem seres humanos.

\section{ANÁLISE DA EXPERIÊNCIA}

\section{Apoio às lactantes diante da pandemia do Covid-19}

Durante o atendimento percebeu-se as lactantes com sentimentos negativos frente à pandemia Covid-19, tais como medo, ansiedade e insegurança. Além das lactantes, algumas vezes, verbalizarem esses sentimentos, também foi possível percebê-los por meio da comunicação não-verbal, quando manifestavam silêncio, choro e expressões faciais de preocupação.

Diante desse contexto psicológico, foi realizada a aplicação da técnica de aconselhamento em saúde em todas as consultas de amamentação, a qual permitiu a compreensão da mãe, a utilização da habilidade de escuta, de tentar apreender o que a mãe está sentindo, que por vezes é difícil verbalizar.

Durante a pandemia, é notória a veiculação constante nos noticiários e mídias sociais de notícias negativas como aumento de contágio, internação e óbitos, que podem inclusive atingir amigos e familiares das lactantes. Notícias como estas podem desencadear medo e ansiedade em qualquer pessoa e, especialmente em lactantes, pois naturalmente estão numa fase de adaptação para um novo momento de suas vidas. Neste sentido, as consultoras orientaram as mulheres a evitarem o excesso de exposição aos noticiários sobre a pandemia, sendo encorajadas a utilizar as mídias sociais para adquirir e trocar informações referentes à maternidade, por isso, as consultoras disponibilizaram seus contatos pessoais para ajudar nesse momento.

Sabendo que os fatores estressantes podem impactar negativamente na amamentação (reflexo da ocitocina), as consultoras criaram estratégias viáveis para dar suporte às lactantes. Foram desenvolvidas lives (encontros online em tempo real) para fornecer informações de qualidade e com embasamento científico para ajudá-las nessa fase da maternidade e sanar as principais dúvidas das famílias. Alguns profissionais da área materno-infantil foram convidados para contribuir na participação das lives, dentre eles: psicólogos, nutricionistas, pediatras, obstetras, fisioterapeutas.

Buscou-se empoderar as mulheres a amamentar esclarecendo que esta é uma forma de proteger seus filhos a partir do oferecimento do alimento padrão ouro, sendo assim, incentivadas a manter a amamentação.

\section{Orientações sobre as medidas de prevenção da infecção do Covid-19}

Durante a anamnese com as mães, percebeu-se que todas estavam amamentando e praticando o distanciamento social preconizado pelas autoridades sanitárias, além dos cuidados básicos de higiene pessoal, como lavagem das mãos, uso de álcool $70 \%$ e banhos mais frequentes. Com o bebê, observou-se que as lactantes mantiveram os cuidados de rotina, mas dando ênfase à limpeza da casa e as restrições de visitas. A restrição de visitas, embora seja uma recomendação para este momento de pandemia, repercute na ausência da rede de apoio (mães, irmãs, avós) tão necessária para algumas mulheres.

As consultoras reforçaram a necessidade de manter esses cuidados e destacaram a importância do acesso a fontes confiáveis, artigos científicos, sites e mídias sociais do MS, secretaria de saúde do estado, SBP e o instagram da empresa de consultoria em amamentação.

Durante os atendimentos, ficou clara a presença de diversas dúvidas em relação às corretas recomendações sobre a prevenção da Covid-19 para a mãe e o bebê, tais como: necessidade de usar máscara mesmo sem sintomas gripais, consequências do uso excessivo do álcool gel e utilização do ar-condicionado no aumento da proliferação da infecção.

Assim, compreendeu-se a necessidade do repasse de informações baseadas nas melhores evidências disponíveis ao que se refere à manutenção do AM.

\section{Manejo da amamentação frente às mudanças}

Percebeu-se que munidas de informações sobre a importância do AM, as lactantes atendidas insistiram em oferecer o leite materno (LM), mesmo diante das adversidades do puerpério imediato, das alterações psicológicas, do cenário da pandemia e da lacuna de rede de apoio presencial, pois estavam em distanciamento social.

Apesar das dificuldades vivenciadas, as mulheres não deixaram de amamentar. No entanto, foram identificados pelas consultoras alguns problemas mamários, como dores e desconfortos, diminuição na produção do leite e ingurgitamento mamário.

O contato presencial com as mulheres que manifestaram problemas mamários nos permitiu praticar técnicas com abordagem preventiva e curativa. Foi acolhido cada relato, as mamas foram avaliadas e foram concedidas orientações sobre o manejo correto diante da situação: ajuste de pega e posição 
correta visando aliviar as dores, massagens circulares iniciando pela aréola e ordenha de alívio para retirada do leite em casos de ingurgitamento, orientações para evitar absorvente mamário e conchas devido a possível proliferação de microorganismos causadores de desconforto, uso de protocolos de ordenha, cuidados com a ingesta hídrica e alimentação da lactante em casos de baixa produção de LM, sendo tudo demonstrado de maneira prática e detalhada.

\section{Importância da consultoria perante a pandemia}

Após os atendimentos, foi possível perceber a satisfação das lactantes pelo serviço prestado, pelo sentimento de empatia, apoio, acolhimento e segurança transmitido às suas famílias nesse momento delicado, principalmente devido estar com a rede de apoio reduzida.

Como consultoras em amamentação foi gratificante e prazeroso receber esse retorno positivo das mães, pois demonstra que o trabalho exaustivo diante da pandemia com o rigoroso cuidado e paramentação adequada trazem resultados positivos para a saúde materno-infantil.

Em nenhum dos atendimentos presenciais houve, por parte das lactantes e das consultoras, qualquer medo do contágio do vírus, pois acreditávamos que a paramentação era suficiente para sua proteção. O mais difícil durante os atendimentos nessa pandemia foi a ausência de toques afetivos, como abraços à família atendida, acarinhar os bebês, limitando-se apenas aos gestos de despedidas e cuidados essenciais.

A prática das enfermeiras consultoras faz parte de um processo interativo, no qual se desenvolve o vínculo com as pacientes, e isso inclui considerar seu aspecto emocional. É fato que a pandemia Covid-19 está gerando inúmeras consequências psicológicas nas pessoas. Pesquisa que analisou 17.865 postagens de usuários evidenciou que as emoções negativas e a sensibilidade social aumentaram, enquanto as emoções positivas e de satisfação diminuíram, sendo imprescindível a criação de políticas que visem intervir precocemente na área da saúde mental. ${ }^{10}$

Diversos países têm se preocupado em desenvolver estratégias que abordem os cuidados em saúde mental para capacitar a sociedade e diminuir o pânico entre outros transtornos mentais. Especialistas da Universidade de Pequim fizeram sugestões de medidas que podem ser eficazes, dentre elas: avaliar a precisão das informações divulgadas, aprimorar os sistemas de apoio social (família, amigos) e utilizar serviços psicossociais, dando ênfase ao aconselhamento por telefone e pela internet com profissionais de saúde. ${ }^{11}$

Estudo qualitativo que também avaliou os sentimentos das mulheres em época de epidemia do Zika Vírus demonstrou relatos de sentimentos de preocupação, medo e insegurança em contrair a doença, bem como em gerar danos ao seu bebê. ${ }^{12}$

Para promover o apoio emocional às lactantes, foi utilizada, em todos os atendimentos, a técnica de aconselhamento em saúde, a qual permitiu utilizar a empatia, o respeito ao tempo da mãe, de compreender o contato visual, a postura, os gestos, a confiança e o interesse, sempre usando uma linguagem sugestiva. $\mathrm{O}$ aconselhamento em saúde é um grande aliado do atendimento durante a amamentação tendo em vista seu efeito positivo nessa prática. Ensaio clínico randomizado controlado realizado com 300 mulheres na Índia o qual avaliou o efeito de um aconselhamento e apoio realizado por conselheiros treinados em amamentação durante os seis primeiros meses de vida da criança, evidenciou o início do AM dentro de uma hora após o parto em 73,4\% no grupo intervenção, em comparação com 33,6\% no grupo controle. Em relação ao AME, mães do grupo intervenção apresentaram taxas maiores $(88,2 \%)$ quando comparadas ao grupo controle (50\%) (OR 7,44; IC 95\% IC: 3,98-13,92). ${ }^{13}$

No enfrentamento da situação de emergência em saúde pública decorrente do Covid-19, o MS publicou a NOTA TÉCNICA № 7/2020-DAPES/SAPS/MS que orienta, conforme a Organização Mundial de Saúde (OMS), a manutenção da amamentação por falta de elementos que comprovem que o leite materno possa disseminar o SARSCoV-2 e devido a seus benefícios para o bebê (MS, 2020). Em casos de mães portadoras do Covid-19, além de manter o AM, o Royal College of Obstetricians and Gynaecologists (RCOG), enfatiza a necessidade de precauções necessárias como o uso de máscara pela lactante e lavagem das mãos antes das mamadas..$^{8,14}$

Diante dos avanços tecnológicos envolvendo compartilhamento de informações é responsabilidade dos profissionais de saúde a disseminação de informações verídicas e cientificamente comprovadas e divulgadas por fontes confiáveis. Diversas organizações de saúde vêm criando intervenções para o controle das Fake News sobre a pandemia Covid-19. No Brasil, o MS criou um canal de WhatsApp para sanar as dúvidas da população, mostrando notícias divulgadas que já foram constatadas como falsas, bem como disponibilizando sites de fontes confiáveis, tais como: o da Agência Nacional de Vigilância Sanitária, Organização Pan-Americana da Saúde, World Health Organization, Biblioteca Virtual em Saúde e o próprio MS. ${ }^{15}$

É sabido que o leite materno é indiscutivelmente o melhor alimento para a criança, gerando impactos positivos na saúde materno-infantil. No entanto, amamentar não é uma habilidade totalmente instintiva do ser humano e precisa ser aprendida e desenvolvida e, portanto, é fundamental o suporte dos profissionais de saúde e da rede social de apoio, pois sem isso o desmame pode ser mais precoce. Diante da pandemia de Covid-19 e da rápida disseminação do vírus, a maioria das lactantes estava cumprindo as recomendações da OMS de manter o distanciamento social. Dessa forma, a rede de apoio se resumia a orientações de amigos e familiares por telefone ou aplicativos de mensagens. ${ }^{16}$

Nesse contexto, e em meio às dificuldades, surgiram alguns problemas mamários. Acredita-se que as queixas referidas estejam relacionadas aos aspectos emocionais relatados durante os atendimentos, pois no processo de amamentar as motivações psíquicas são fatores que podem interferir nos hormônios envolvidos na amamentação. Ao se analisar a relação entre estresse pós-traumático e os níveis de ocitocina há evidências de que mulheres com estresse pós-traumático elevado apresentam 
níveis mais baixos de ocitocina $(r(8): 0,77 ; p: 0,026)$, sendo imprescindível que enfermeiras apoiem e incentivem a lactação em mães que sofreram estresse pós-traumático. ${ }^{17}$

A atuação das enfermeiras consultoras foi fundamental para a promoção do AM mesmo no atual contexto vivido. Evidências analisadas a partir de uma revisão sistemática apontaram que intervenções realizadas por consultores e conselheiros da amamentação aumentam a prevalência de mulheres iniciando o AM (OR:1,35; IC95\%:1,10-1,67), bem como melhoram as taxas de amamentação com um mês (OR:1,49; IC95\%:1,09-2,04) e as taxas de AME (OR:1,71; IC95\%:1,20-2,44). ${ }^{18}$

No cenário da pandemia, as mulheres se viram prejudicadas pela falta da rede de apoio, o que se tornou um fator preocupante, pois a presença física da família ocupa o primeiro lugar na referência das mulheres brasileiras. Ter uma pessoa da família por perto viabiliza dois aspectos peculiares: os cuidados ministrados pelos familiares aos recém-nascidos e o apoio à própria puérpera, dando sensação de força e melhora da autoestima. ${ }^{19}$

Nesse sentido, as consultoras buscaram estratégias de ação semelhantes ao Sistema Único de Saúde do Brasil que estão sendo realizadas diante da pandemia: difundir informações sobre os cuidados na prevenção do Covid-19, incentivar o autocuidado, promover visitação virtual e, em casos necessários, garantir cuidado em condições seguras no atendimento presencial. ${ }^{20}$

Respeitando o distanciamento social recomendado, o principal meio utilizado pelas consultoras para os atendimentos foi a mídia social, a qual é amplamente reconhecida no âmbito da promoção do AM, gerando repercussões positivas, levando informações ao público-alvo, promovendo mudanças de comportamentos e otimizando, com custo baixo e acessível à população.

Diante da pandemia Covid-19 percebeu-se o quão exaustivo tem sido para as consultoras prestarem o cuidado assistencial. Recente revisão evidenciou sofrimento psicológico entre profissionais de saúde expostos à Covid-19 devido à exposição a altos níveis de eventos estressantes, sendo também importante o cuidado à saúde mental dos trabalhadores por meio da telemedicina. ${ }^{21}$

\section{CONCLUSÕES E IMPLICAÇÕES PARA A PRÁTICA}

No contexto da Pandemia Covid-19, a consultoria em amamentação constitui-se como um dispositivo agregador na atenção à saúde das mulheres que favorece tanto a promoção do AM quanto a saúde mental das lactantes. Na presença de sentimentos psicológicos negativos, foi possível oferecer apoio por meio do aconselhamento, bem como orientações com equipe multiprofissional.

Assim como em outros serviços, as mídias sociais foram essenciais para manter o cuidado a essas lactantes frente a recomendação do distanciamento social. Diante da lacuna das redes de apoio, as consultoras buscaram promover maior segurança às mulheres por meio de acolhimento e orientações. Vislumbra-se a manutenção de tais atividades após a pandemia.
Pontua-se o quão importante é a disponibilização de informações confiáveis para embasar as mães, pois além das dificuldades comuns ao puerpério, elas vivenciavam o cenário crítico da pandemia, em que notícias falsas, negativas e excessivas podem prejudicar expressivamente a saúde mental das lactantes, e consequentemente, o processo de amamentar.

Pode-se citar como limitação do estudo o relato envolver apenas a percepção das consultoras, e não das mães atendidas. Porém, o relato desta experiência traz informações e direcionamentos para uma prática holística dos profissionais que apoiam a amamentação, com vistas à melhoria da qualidade do cuidado prestado. A divulgação e o consumo crítico dessa experiência são imprescindíveis para os atuais desafios da promoção da saúde diante da pandemia e pode fomentar novas estratégias exitosas.

\section{CONTRIBUIÇÕES DOS AUTORES}

Desenho do relato de experiencia. Anne Fayma Lopes Chaves. Mariana Gonçalves de Oliveira. Sabrina Alapenha Ferro Chaves Costa Lima.

Levantamento informações. Anne Fayma Lopes Chaves. Mariana Gonçalves de Oliveira.

Análise de dados. Ana Carolina Maria Araújo Chagas Costa Lima. Sabrina Alapenha Ferro Chaves Costa Lima. Mônica Oliveira Batista Oriá.

Interpretação dos resultados. Ana Carolina Maria Araújo Chagas Costa Lima. Mariana Gonçalves de Oliveira. Márcia Maria Tavares Machado. Mônica Oliveira Batista Oriá.

Redação e revisão crítica do manuscrito. Ana Carolina Maria Araújo Chagas Costa Lima. Anne Fayma Lopes Chaves. Mariana Gonçalves de Oliveira. Sabrina Alapenha Ferro Chaves Costa Lima. Márcia Maria Tavares Machado. Mônica Oliveira Batista Oriá.

Aprovação da versão final do artigo. Ana Carolina Maria Araújo Chagas Costa Lima. Anne Fayma Lopes Chaves. Mariana Gonçalves de Oliveira. Sabrina Alapenha Ferro Chaves Costa Lima. Márcia Maria Tavares Machado. Mônica Oliveira Batista Oriá.

Responsabilidade por todos os aspectos do conteúdo e a integridade do artigo publicado. Ana Carolina Maria Araújo Chagas Costa Lima. Anne Fayma Lopes Chaves. Mariana Gonçalves de Oliveira. Sabrina Alapenha Ferro Chaves Costa Lima. Márcia Maria Tavares Machado. Mônica Oliveira Batista Oriá.

\section{EDITOR ASSOCIADO}

Antonio José Almeida Filho

\section{REFERÊNCIAS}

1. International Board of Lactation Consultant Examiners. Position paper on the role and impact of the IBCLC [Internet]. 2020 [citado 2 out 2020]. Disponivel em: https://iblce.org/

2. Chaves AFL, Vitoriano LNH, Borges FLP, Melo RDA, Oliveira MG, Costa Lima ACMAC. Percepção das mulheres que receberam consultoria em amamentação. Enferm. Foco. 2019;10(5):79-84. http://dx.doi. org/10.21675/2357-707X.2019.v10.n5.2519.

3. Ministério da Saúde (BR). Parecer do Conselho Federal de Enfermagem no 18/2016/CTAS. Solicitação de parecer a respeito de atividades de 
consultoria em amamentação [Internet]. 2020 [citado 18 maio 2020]. Disponível em: http://www.cofen.gov.br/parecer-no-182016ctas_47897. $\mathrm{html}$

4. Worldometer. Real time world statistics [Internet]. 2020 [citado 23 jul 2020]. Disponível em: https://www.worldometers.info/coronavirus/

5. Shanes ED, Mithal LB, Otero S, Azad HA, Miller ES, Goldstein JA Placental pathology in COVID-19. Am J Clin Pathol. 2020 jul;154(1):2332. http://dx.doi.org/10.1093/ajcp/aqaa089.

6. Chen H, Guo J, Wang C, Luo F, Yu X, Zhang W et al. Clinical characteristics, and intrauterine vertical transmission potential of COVID-19 infection in nine pregnant women: a retrospective review of medical records. Lancet. 2020;6736(10226):1-7. http://dx.doi.org/10.1016/S0140 6736(20)30360-3.

7. De Rose DU, Piersigilli F, Ronchetti MP, Santisi A, Bersani I, Dotta A et al. Novel Coronavirus disease (COVID-19) in newborns and infants: what we know so far. Ital J Pediatr. 2020;46(56):1-8. http://dx.doi.org/10.1186/ s13052-020-0820-x.

8. Royal College of Obstetricians \& Gynaecologists. Coronavirus (COVID-19) infection in pregnancy: information for healthcare professionals. Version 10 [Internet] 2020 [citado 23 jul 2020]. Disponível em: https://www.rcog org.uk/globalassets/documents/guidelines/2020-06-04-coronaviruscovid-19-infection-in-pregnancy.pdf

9. Daltro MR, Faria AA. Experience report: a scientific narrative in the post-modernity. Estud Pesqui Psicol. 2019;19(1):223-37. http://dx.doi. org/10.12957/epp.2019.43015.

10. Li S, Wang Y, Xue J, Zhao N. Zhu, T. The Impact of COVID-19 Epidemic declaration on psychological consequences: a study on active weibo users. Int J Environ Res Public Health. 2020;17(2032):1-9. http://dx. doi. org/10.3390/ijerph17062032.

11. BaoY, SunY, Meng S, Shi J, Lu L. 2019-nCoV epidemic: address mental health care to empower society. Lancet. 2020;395(10224):37-8. http:// dx.doi.org/10.1016/S0140-6736(20)30309-3.

12. Silva FWO, Roscoche KGC, Farias RJO, Abreu LAF, Sousa AAS, Chaves AFL. Zika virus: feelings and practices of caregivers. Rev Enferm UFSM. 2020;10:e9. https://doi.org/10.5902/2179769239258.
13. Gupta A, Dadhich JP, Ali SM, Thakur N. Skilled counseling in enhancing early and exclusive breastfeeding rates: an experimental study in an urban population in India. Indian Pediatr. 2019;56(2):114-8. http://dx.doi. org/10.1007/s13312-019-1482-x.

14. Ministério da Saúde (BR), Secretaria de Atenção Primária à Saúde Departamento de Ações Programáticas Estratégicas, CoordenaçãoGeral de Ciclos da Vida, Coordenação de Saúde das Mulheres. Nota Técnica $n^{0}$ 7/2020. Atenção às gestantes no contexto da infecção covid-19 causada pelo novo coronavírus (SARS-CoV-2). Brasília: Ministério da Saúde; 2020.

15. Conselho Regional de Farmácia do Estado de São Paulo. Manual de orientação ao farmacêutico: COVID-19. São Paulo: Conselho Regional de Farmácia do Estado de São Paulo; 2020.

16. Ministério da Saúde (BR), Secretaria de Vigilância em Saúde, Centro de Operações de Emergências em Saúde Pública. Especial: doença pelo coronavírus 2019. Brasília: Ministério da Saúde; 2020. (Boletim Epidemiológico, no. 7).

17. Garfield L, Holditch-Davis D, Carter CS, McFarlin BL, Seng JS, Giurgescu $\mathrm{C}$ et al. A pilot study of oxytocin in low-income women with a low birth-weight infant: is oxytocin related to posttraumatic stress? Adv Neonatal Care. 2019;19(4):12-21. http://dx.doi.org/10.1097/ ANC.0000000000000601.

18. Patel S, Patel S. The effectiveness of lactation consultants and lactation counselors on breastfeeding outcomes. J Hum Lact. 2016;32(3):530-41. http://dx.doi.org/10.1177/0890334415618668

19. Lima SP, Santos EKA, Erdmann AL, Souza AIJ. Unveiling the lived experience meaning of being a woman breastfeeding with puerperal complications. Texto Contexto Enferm. 2017;26(3):e0880016. http:// dx.doi.org/10.1590/0104-07072018000880016.

20. Facchini L. COVID-19: Nocaute do neoliberalismo? Será possível fortalecer os princípios históricos do SUS e da APS em meio à pandemia? APS em Revista. 2020;2(1):3-10. http://dx.doi.org/10.14295/aps.v2i1.73.

21. Talevi D, Socci V, Carai M, Carnaghi G, Faleri S, Trebbi E et al. Mental health outcomes of the COVID-19 pandemic. Riv Psichiatr. 2020;55(3):13744. http://dx.doi.org/10.1708/3382.33569. 